\title{
Proceeding Paper \\ Stimulated Raman Adiabatic Passage in a Quantum Emitter Near to a Gold Nanoparticle
}

\author{
Natalia Domenikou ${ }^{1}$, Ioannis Thanopulos ${ }^{1}\left[\right.$, Vassilios Yannopapas $^{2}\left(\mathbb{D}\right.$ ) and Emmanuel Paspalakis ${ }^{1, *(1)}$ \\ 1 Materials Science Department, School of Natural Sciences, University of Patras, 26504 Patras, Greece; \\ domenikou.n@gmail.com (N.D.); ithano@upatras.gr (I.T.) \\ 2 Department of Physics, National Technical University of Athens, 15780 Athens, Greece; \\ vyannop@mail.ntua.gr \\ * Correspondence: paspalak@upatras.gr
}

check for updates

Citation: Domenikou, N.; Thanopulos, I.; Yannopapas, V.; Paspalakis, E. Stimulated Raman Adiabatic Passage in a Quantum Emitter Near to a Gold Nanoparticle. Mater. Proc. 2021, 4, 7. https:// doi.org/10.3390/IOCN2020-07867

Academic Editors: Ana María

Díez-Pascual, Antonio Di Bartolomeo and Guanying Chen

Published: 11 November 2020

Publisher's Note: MDPI stays neutral with regard to jurisdictional claims in published maps and institutional affiliations.

Copyright: (c) 2020 by the authors Licensee MDPI, Basel, Switzerland. This article is an open access article distributed under the terms and conditions of the Creative Commons Attribution (CC BY) license (https:// creativecommons.org/licenses/by/ $4.0 /)$.

\begin{abstract}
In this work, we present numerical results on the influence of a spherical metallic nanoparticle to the population transfer in a $\Lambda$-type quantum system under conditions of Stimulated Raman Adiabatic Passage (STIRAP). For the study of the system's dynamics, we use the density matrix approach for the quantum system, where the parameters for the electric field amplitudes and the spontaneous decay rates have been calculated using ab initio electromagnetic calculations for the plasmonic nanoparticle. We present results for the evolution of the populations of the different levels of the quantum system as a function of different parameters, in the presence and the absence of the plasmonic nanoparticle. We find that the presence of the plasmonic nanoparticle and the polarization of the pump and Stokes fields with respect to the surface of the nanoparticle, affect the efficiency of the population transfer inside the three-level quantum system. For the right combination of the values of the modified spontaneous decay rates and the fields intensities, high efficiency population transfer is obtained in the quantum system near a plasmonic nanoparticle using STIRAP process.
\end{abstract}

Keywords: Stimulated Raman Adiabatic Passage technique (STIRAP); $\Lambda$-type three-level quantum system; metallic nanoparticle; coherent population transfer

\section{Introduction}

The last three decades, Stimulated Raman Adiabatic Passage (STIRAP) [1] has been proven a robust and high-efficient technique for population transfer in a three-level quantum system and beyond that. As coupled quantum-plasmonic nanostructures are widely used in recent nanophotonics for the superior properties that the coupled structures have over their constituents, a series of studies have analyzed the influence of a spherical metallic nanoparticle (MNP), which is a basic plasmonic nanosystem, on coherent population transfer methods in nearby quantum systems [2-8].

For several recent proposals, it is important to understand the behavior of STIRAP near MNPs. Therefore, in this work we present numerical results on the influence of a spherical MNP to the population transfer in a $\Lambda$-type quantum system, a model semiconductor quantum dot (SQD), under conditions of STIRAP. For the study of the system's dynamics, we use the density matrix approach for the quantum system, where the parameters for the electric field amplitudes and the spontaneous decay rates have been calculated using $a b$ initio electromagnetic calculations for the plasmonic nanoparticle [9]. We then present results for the evolution of the populations of the different levels of the quantum system as a function of different parameters, in the presence and the absence of the plasmonic nanoparticle. We find that the presence of the MNP and the polarization of the pump and Stokes fields with respect to the surface of the nanoparticle, affect the efficiency of the population transfer inside the three-level quantum system. For the right combination of the values of the modified spontaneous decay rates and the pump and Stokes fields 
intensities, high efficiency population transfer is obtained in the SQD near a MNP using STIRAP technique.

\section{Theory}

The model system under study consists of a spherical MNP with radius $R=50 \mathrm{~nm}$, and a $\Lambda$-type three-level quantum system with transition energies $\hbar \omega_{01}=\hbar \omega_{02}=1.517 \mathrm{eV}$, as shown in Figure 1a. We allow each laser pulse to drive only one transition.
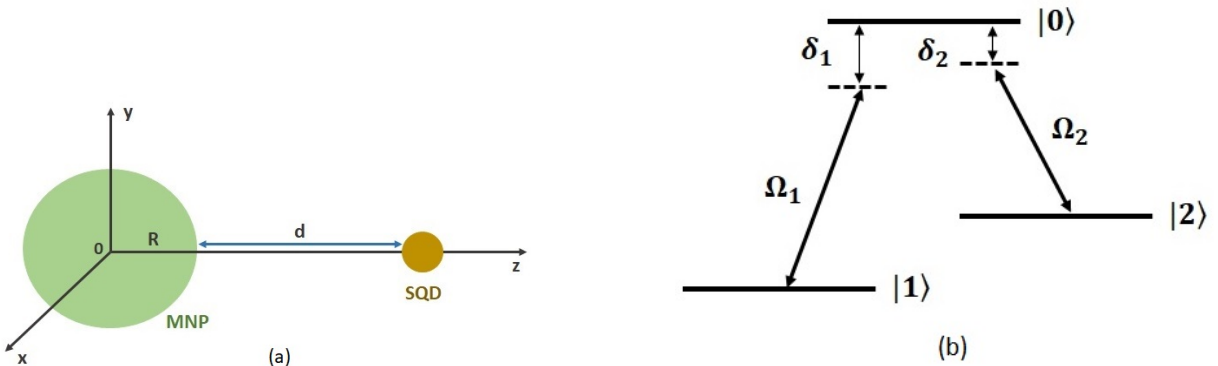

(b)
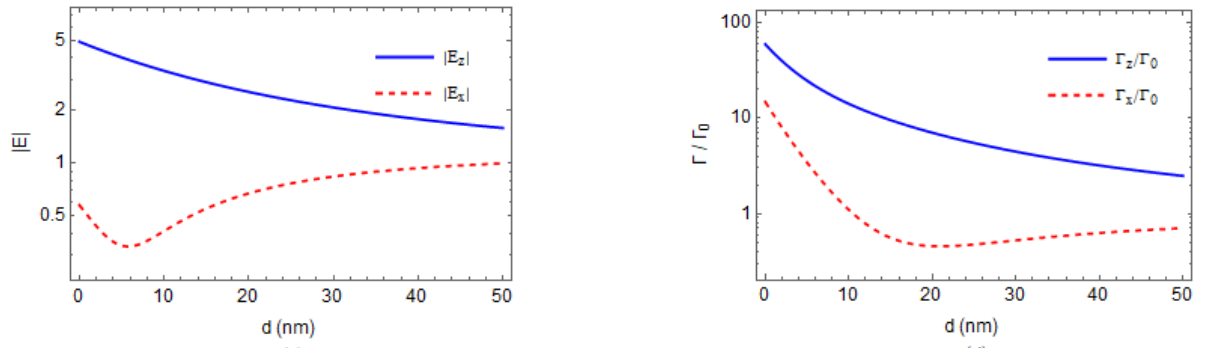

(d)

Figure 1. The SQD at distance $d$ from the surface of a MNP with radius $R$ (a) and schematic representation of the three-level $\Lambda$-type model $(\mathbf{b})$ with $\Omega_{n}$ and $\delta_{n},(n=1,2)$, standing for the Rabi frequencies and detunings of the $|n\rangle \rightarrow|0\rangle$ laser-induced transitions, respectively. The modified field factor (c) and the modified spontaneous decay rate, the Purcell factor, (d) along $z$ and $x$ axis with respect to the surface of the MNP.

The electric field is described classically by $\vec{E}(t)=\hat{\varepsilon}_{1} \mathcal{E}_{1} f_{1}(t) e^{i \bar{\omega}_{1} t}+\hat{\varepsilon}_{2} \mathcal{E}_{2} f_{2}(t) e^{i \bar{\omega}_{2} t}+$ c.c., where $\hat{\varepsilon}_{n}$ is the polarization vector, $\mathcal{E}_{n}$ is the electric field amplitude and $f_{n}(t)$ is the dimensionless pulse envelope of each laser pulse. We use Gaussian pulses with $f_{1}(t)=$ $e^{-\left(t-t_{0}-t_{n}\right)^{2} /\left(2 t_{p}^{2}\right)}$ and $f_{2}(t)=e^{-\left(t-t_{0}\right)^{2} /\left(2 t_{p}^{2}\right)}$, where $t_{0}$ is the common central time of the two pulses, $t_{p}$ characterizes the pulse duration and $t_{n}$ is the time-delay between the two applied fields. In our system we have $t_{p}=1 \mathrm{~ns}, t_{n}=2 \mathrm{~ns}$ and $t_{0}=4 \mathrm{~ns}$. We note that positive $t_{n}$ leads to the counter-intuitive pulse sequence for population transfer from state $|1\rangle$ to $|2\rangle$, which is characteristic of the STIRAP technique $[1,10]$. The angular frequency of the pump field is $\bar{\omega}_{1}$ and of the Stokes field is $\bar{\omega}_{2}$. We consider that the fields are polarized either tangentially, i.e., along the $x$ axis, or radially, i.e., along the $z$ axis, with respect to the surface of the MNP.

The Hamiltonian of the laser-matter interaction in this system, within the rotating wave and dipole approximations, is given by $\hat{H}(t)=\Omega_{1}(t) e^{-i \delta_{1} t}|1\rangle\left\langle 0\left|+\Omega_{2}(t) e^{-i \delta_{2} t}\right| 2\right\rangle\langle 0|+$ c.c. with $\hbar=1$. Here, $\Omega_{n}(t)=\Omega_{n}^{0} f_{n}(t)$ with $\Omega_{n}^{0}(t)=-\vec{\mu}_{n 0} \cdot \vec{\varepsilon}_{n} \mathcal{E}_{n} f_{n}(t)$, with $n=1,2$, is the Rabi frequency of the transition $|n\rangle \leftrightarrow|0\rangle$, with $\vec{\mu}_{n 0}$ being the associated dipole transition matrix element. Also, $\delta_{n}=\omega_{0}-\omega_{n}-\bar{\omega}_{n}=\omega_{0 n}-\bar{\omega}_{n}$ is the laser field detuning from resonance for the transition $|n\rangle \leftrightarrow|0\rangle$, with the energies of the n-th lower level and upper level, respectively, being $\omega_{n}$ and $\omega_{0}$. 
In all the calculations presented below, we take the laser fields at exact resonance with the adjacent transitions, i.e., $\delta_{1}=\delta_{2}=0$. The equations of motion of the density matrix elements are given by

$$
\begin{aligned}
& i \dot{\rho}_{00}(t)=-i\left(\Gamma_{01}+\Gamma_{02}\right) \rho_{00}(t)+\Omega_{1}^{*}(t) \rho_{10}(t)-\Omega_{1}(t) \rho_{01}(t)+\Omega_{2}^{*}(t) \rho_{20}(t)-\Omega_{2}(t) \rho_{02}(t), \\
& i \dot{\rho}_{11}(t)=i \Gamma_{01} \rho_{00}(t)+\Omega_{1}(t) \rho_{01}(t)-\Omega_{1}^{*} \rho_{10}(t) \\
& i \dot{\rho}_{22}(t)=i \Gamma_{02} \rho_{00}(t)+\Omega_{2}(t) \rho_{02}(t)-\Omega_{2}^{*} \rho_{20}(t) \\
& i \dot{\rho}_{10}(t)=-i \gamma_{10} \rho_{10}(t)+\Omega_{1}(t) \rho_{00}-\Omega_{1}(t) \rho_{11}(t)-\Omega_{2}(t) \rho_{12}(t) \\
& i \dot{\rho}_{20}(t)=-i \gamma_{20} \rho_{20}(t)+\Omega_{2}(t) \rho_{00}-\Omega_{1}(t) \rho_{21}(t)-\Omega_{2}(t) \rho_{22}(t) \\
& i \dot{\rho}_{12}(t)=\Omega_{1}(t) \rho_{02}(t)-\Omega_{2}^{*}(t) \rho_{10}(t)
\end{aligned}
$$

with $\sum_{n} \rho_{n n}(t)=1$ and $\rho_{n m}(t)=\rho_{m n}^{*}(t)$. We have assumed a closed system, i.e., there is no decay to levels outside of the three-level manifold under study. We denote by $\Gamma_{01}\left(\Gamma_{02}\right)$ the spontaneous decay rate from level $|0\rangle$ to level $|1\rangle(|2\rangle)$ with $\Gamma_{01}=\Gamma_{02}=\Gamma_{0}$ and by $\gamma_{n m}$ the coherence decay rate between states $|n\rangle$ and $|m\rangle$, with $\gamma_{10}=\gamma_{20}=\frac{1}{2}\left(\Gamma_{01}+\Gamma_{02}\right)$. We note that we use fourth-order Runge-Kutta method for the numerical solution of the above equations.

\section{Results and Discussion}

In this section, we investigate the influence of the MNP on the target-state population dynamics using the STIRAP technique for various parameters.

In Figure 2, we investigate the time evolution of the population of each state of the $\Lambda$-type system for different distances between the MNP and the SQD. The pump field is polarized along the $x$ axis, with $\Omega_{1}^{0}=10 \mathrm{~ns}^{-1}$, and the Stokes field is polarized along the $z$ axis, with $\Omega_{2}^{0}=1 \mathrm{~ns}^{-1}$. We find that the population transfer in the SQD under the STIRAP technique works with high efficiency for $d<10 \mathrm{~nm}$. Also, we note that the efficiency of population transfer decreases when the SQD-MNP distance is large. As the SQD moves away from the MNP, oscillatory population dynamics is also observed because the adiabatic condition is almost fulfilled. On the other hand, in the absence of the MNP (Figure 2a), we do not obtain high population in the target state of the SQD using STIRAP, due to the insufficient fulfillment of the adiabatic condition.

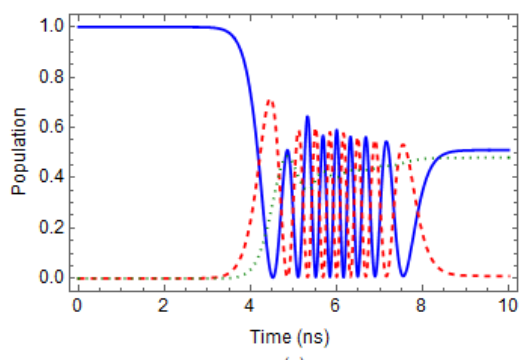

(a)

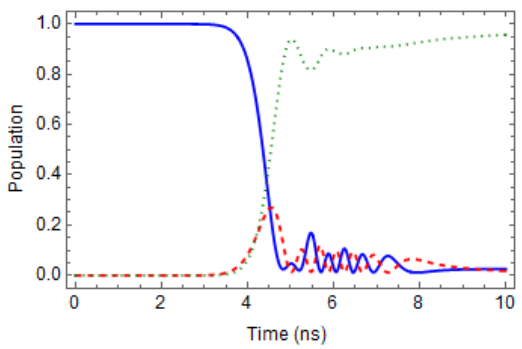

(c)

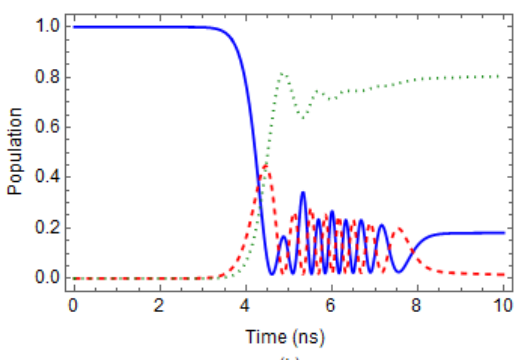

(b)

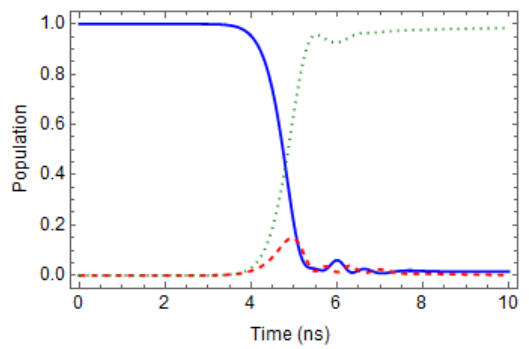

(d)

Figure 2. Cont. 


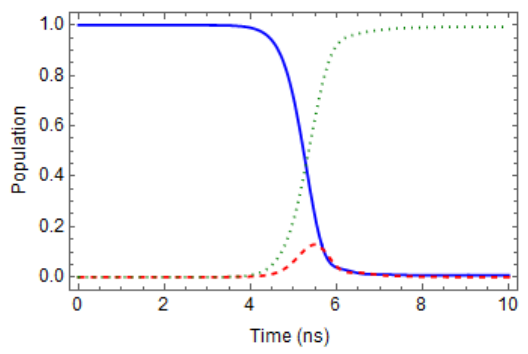

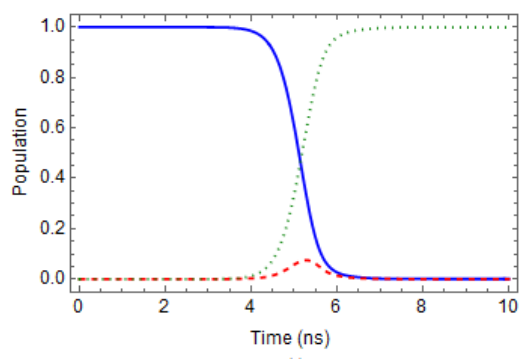

Figure 2. The two columns present the population evolution of $\rho_{11}(t)$ (blue solid curve), $\rho_{00}(t)$ (red dashed curve) and $\rho_{22}(t)$ (green dotted curve) at $d \rightarrow \infty(\mathbf{a}), d=50 \mathrm{~nm}(\mathbf{b}), d=30 \mathrm{~nm}(\mathbf{c}), d=15 \mathrm{~nm}$ (d), $d=5 \mathrm{~nm}(\mathbf{e})$, and $d=1 \mathrm{~nm}(\mathbf{f})$, with $\Gamma_{0}=0.1 \mathrm{~ns}^{-1}$. The polarization direction of the pump field is along the $x$ axis with $\Omega_{1}^{0}=10 \mathrm{~ns}^{-1}$ and of the Stokes field along the $z$ axis with $\Omega_{2}^{0}=1 \mathrm{~ns}^{-1}$.

In Figure 3, we further study the time evolution of the population of each state of the $\Lambda$-type system but for the inverse polarizations of the two applied fields, i.e., the pump field is polarized along the $z$ axis and Stokes field is polarized along the $x$ axis. We use $\Omega_{1}^{0}=1 \mathrm{~ns}^{-1}$ and $\Omega_{2}^{0}=10 \mathrm{~ns}^{-1}$. We find that the population transfer in the SQD under the STIRAP technique works efficiently for $d \leq 10 \mathrm{~nm}$, with transfer efficiency above $80 \%$. In the absence of the MNP, as well as for distances $d>30 \mathrm{~nm}$, we note that the adiabatic condition is not fulfilled, STIRAP does not work properly and the intermediate state $|0\rangle$ has population both in the transient regime and even at the end of the population dynamics.
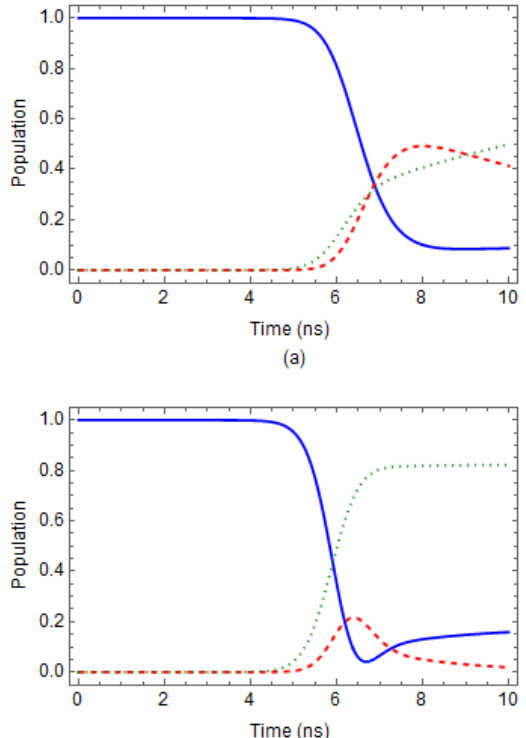

(c)

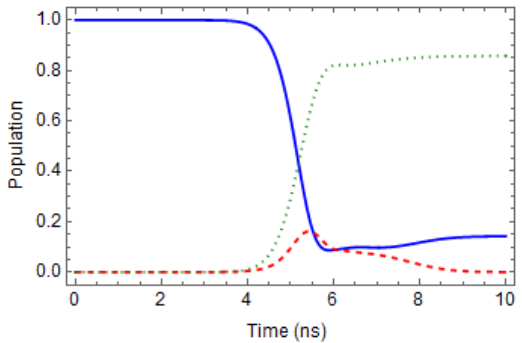

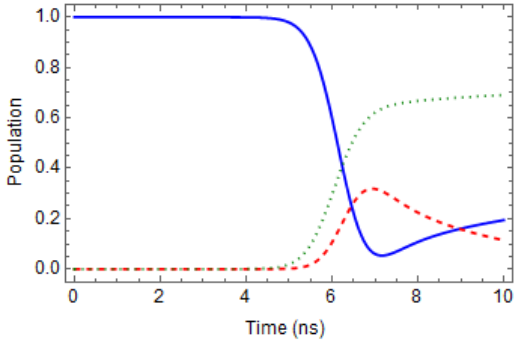

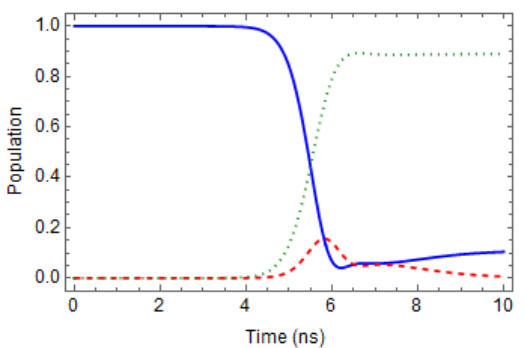

(d)

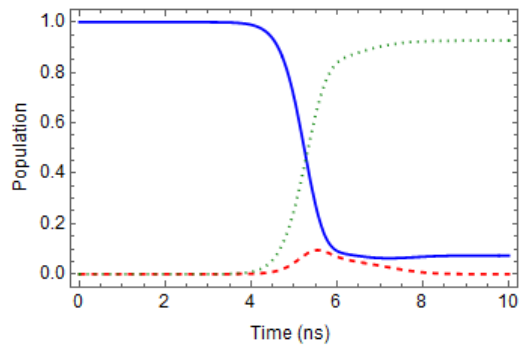

Figure 3. The two columns present the population evolution of $\rho_{11}(t)$ (blue solid curve), $\rho_{00}(t)$ (red dashed curve) and $\rho_{22}(t)$ (green dotted curve) at $d \rightarrow \infty(\mathbf{a}), d=50 \mathrm{~nm}(\mathbf{b}), d=30 \mathrm{~nm}(\mathbf{c}), d=15 \mathrm{~nm}$ $(\mathbf{d}), d=5 \mathrm{~nm}(\mathbf{e})$, and $d=1 \mathrm{~nm}(\mathbf{f})$, with $\Gamma_{0}=0.1 \mathrm{~ns}^{-1}$. The polarization direction of the pump field is along the $z$ axis with $\Omega_{1}^{0}=1 \mathrm{~ns}^{-1}$ and of the Stokes field along the $x$ axis with $\Omega_{2}^{0}=10 \mathrm{~ns}^{-1}$. 


\title{
4. Conclusions
}

In summary, in this work, we have studied how a MNP affects the STIRAP population transfer process in a $\Lambda$-type three-level SQD. We investigated the time evolution of the population of the SQD for different positions between the SQD and the MNP and different polarizations of the pump and Stokes field. In the first case that the pump laser is polarized along the $x$ axis and the Stokes field is polarized along the $z$ axis, the population transfer using STIRAP is highly efficient as the distance between the SQD and the MNP decreases. The same dynamical behavior is observed in the second case, when the applied fields are polarized in opposite way. A difference between the two cases is that in the second case, we do not observe oscillations in the population dynamics. Also, the population transfer efficiency is higher in the first case for this pair of parameters. We conclude that for suitable values of the above parameters the STIRAP technique is a robust high-efficient population transfer process for a SQD near a MNP and the presence of the MNP may help the efficiency of STIRAP. Our results can be of importance for applications of quantum photonic devices in nanotechnology.

Author Contributions: Conceptualization, E.P.; methodology, N.D., V.Y., I.T. and E.P.; software, N.D., V.Y. and I.T.; validation, N.D., V.Y., I.T. and E.P.; investigation, N.D., I.T. and E.P.; writingoriginal draft preparation, N.D., I.T. and E.P.; writing-review and editing, N.D., I.T. and E.P.; visualization, N.D.; supervision, I.T. and E.P. All authors have read and agreed to the published version of the manuscript.

Conflicts of Interest: The authors declare no conflict of interest.

\author{
Abbreviations \\ The following abbreviations are used in this manuscript: \\ STIRAP Stimulated Raman Adiabatic Passage \\ MNP metallic nanoparticle \\ SQD semiconductor quantum dot
}

\section{References}

1. Vitanov, N.V.; Rangelov, A.A.; Shore, B.W.; Bergmann, K. Stimulated Raman adiabatic passage in physics, chemistry, and beyond. Rev. Mod. Phys. 2017, 89, 015006.

2. Sadeghi, S.M. The inhibition of optical excitations and enhancement of Rabi flopping in hybrid quantum dot-metallic nanoparticle systems. Nanotechnology 2009, 20, 225401.

3. Sadeghi, S.M. Tunable nanoswitches based on nanoparticle meta-molecules. Nanotechnology 2010, $21,355501$.

4. Antón, M.A.; Carreño, F.; Melle, S.; Calderón, O.G.; Cabrera-Granado, E.; Cox, J.; Singh, M.R. Plasmonic effects in excitonic population transfer in a driven semiconductor-metal nanoparticle hybrid system. Phys. Rev. B 2012, 86, 155305.

5. Paspalakis, E.; Evangelou, S.; Terzis, A.F. Control of excitonic population inversion in a coupled semiconductor quantum dot-metal nanoparticle system. Phys. Rev. B 2013, 87, 235302.

6. Yang, W.X.; Chen, A.X.; Huang, Z.; Lee, R.K. Ultrafast optical switching in quantum dot-metallic nanoparticle hybrid systems. Opt. Express 2015, 23, 13032-13040.

7. McMillan, R.J.; Stella, L.; Grüning, M. Projected equations of motion approach to hybrid quantum/classical dynamics in dielectric-metal composites. Phys. Rev. B 2016, 94, 125312.

8. Qi, Y.; Shu, C.-C.; Dong, D.-Y.; Petersen, I.R.; Jacobs, K.; Gong, S. Fast quantum state transfer in hybrid quantum dot-metal nanoparticle systems by shaping ultrafast laser pulses. J. Phys. D Appl. Phys. 2019, 52, 425101.

9. Carreño, F.; Antón, M.A.; Yannopapas, V.; Paspalakis, E. Resonance fluorescence spectrum of a $\Lambda$-type quantum emitter close to a metallic nanoparticle. Phys. Rev. A 2016, 94, 013834.

10. Kral, P.; Thanopulos, I.; Shapiro, M. Colloquium: Coherently controlled adiabatic passage. Rev. Mod. Phys. 2007, 79, 53. 